\title{
Use of CT-guided periradicular injection for the treatment of foraminal and extraforaminal disc herniations
}

\author{
Authors Marcelo F Gruenberg, Matías Petracchi, Marcelo Valacco, Carlos Solá \\ Institution Hospital Italiano of Buenos Aires, Potosi 4215, Buenos Aires, Argentina
}

\begin{tabular}{|c|c|}
\hline $\begin{array}{l}\text { Final Class of evidence } \\
\text { (CoE)-treatment }\end{array}$ & Yes \\
\hline \multicolumn{2}{|l|}{ Study design: } \\
\hline \multicolumn{2}{|l|}{ RCT } \\
\hline \multicolumn{2}{|l|}{ Cohort } \\
\hline \multicolumn{2}{|l|}{ Case control } \\
\hline Case series & - \\
\hline \multicolumn{2}{|l|}{ Methods } \\
\hline \multicolumn{2}{|l|}{ Concealed allocation (RCT) } \\
\hline \multicolumn{2}{|l|}{ Intention to treat (RCT) } \\
\hline \multicolumn{2}{|l|}{$\begin{array}{l}\text { Blinded/independent } \\
\text { evaluation of primary outcome }\end{array}$} \\
\hline $\mathrm{F} / \mathrm{U} \geq 85 \%$ & - \\
\hline Adequate sample size & - \\
\hline \multicolumn{2}{|l|}{ Control for confounding } \\
\hline Overall class of evidence & IV \\
\hline \multicolumn{2}{|c|}{$\begin{array}{l}\text { The definiton of the different } \\
\text { classes of evidence is available on } \\
\text { page } 59 \text {. }\end{array}$} \\
\hline
\end{tabular}

\section{ABSTRACT}

Study design: Retrospective case series. Evidence level IV.

Objectives: To evaluate surgical candidates with foraminal or extraforaminal lumbar disc herniation treated with CT-guided periradicular injection (CTGPI) as a valid treatment option for avoiding surgery.

Methods: We carried out a retrospective evaluation of 46 consecutive patients with foraminal or extraforaminal disc herniation treated with CTGPI. CTGPI was performed only when radicular pain could not be controlled, or in patients who continued requiring pain medication following an acute episode and whose radicular pain precluded them from resuming their daily activities. Forty-six patients with a minimum 2-year follow-up met the inclusion criteria. There were 21 women and 25 men, with a mean age of 47 years.

Results: At 1 month after injection, 41 (89\%) patients experienced a decrease in radicular pain; 3 experienced no change; and 2 had received surgical treatment. At the final follow-up visit (mean, 74 months) 6 additional patients underwent surgery while 38 (83\%) did not require surgery. Pain level comparison between pre-injection and last examination showed that low back pain had decreased a mean of 5 points and radicular pain diminished a mean of 7 points. Twenty-two $(58 \%)$ of the 38 nonoperated patients had no pain at all and 35 patients had resumed their normal daily activities. No complications were recorded.

Conclusion: Based on these results, we consider that the use of CTGPI is a reliable alternative before surgery for patients with foraminal or extraforaminal disc herniation without severe motor deficit but with intractable radicular pain.

The authors have no conflict of interest for this study. No funding was received for research. 


\section{STUDY RATIONALE}

Peridural steroid injections appear as a treatment of unreliable result. Most patients with lumbar radicular compressions received surgical treatment as the last treatment option for the management of severe sciatica or cruralgia resistant to conservative therapy. Many patients would be able to avoid surgery if these symptoms could be controlled with a conservative treatment.

We believe that peridural injections could be a good treatment option to avoid surgery.

\section{OBJECTIVES}

The purpose of this study is to evaluate surgical candidates with foraminal or extraforaminal lumbar disc herniation treated with CT-guided periradicular injection (CTGPI) as a valid treatment option to avoid surgery.

\section{METHODS}

Study design: Retrospective case series. Evidence level IV.

Inclusion criteria: Patients with sciatica or cruralgia of at least 4 weeks duration resistant to conventional medical therapy, considered by providers for surgical treatment, with a clear diagnosis of foraminal or extraforaminal disc herniation.

\section{Exclusion criteria (Fig 1):}

- Other pathologies that could produce similar symptoms

- Central or foraminal stenosis

- Previous lumbar surgery

- Clinical depression

- Anticoagulation treatment

- Pregnancy

- Metabolic radiculopathies

\section{Patient population and interventions (Fig 1):}

- From January 2000 to April 2003, 1542 CT-guided nerve root injections were performed in our institution. We carried out a retrospective evaluation of 46 consecutive-selected patients with foraminal or extraforaminal disc herniation treated with CTGPI during this period.
- All patients have at least 2 years of follow-up.

- Before the periradicular injection, all patients underwent a complete trial of conventional medical therapy including bed rest, physiotherapy, antiinflammatory drugs, and analgesics.

- CTGPI was performed only when radicular pain could not be controlled or in patients who continued requiring pain medication following an acute episode and whose radicular pain precluded them from resuming their daily activities.

- Forty-six patients with a minimum 2-year follow-up met the inclusion criteria. There were 21 women and 25 men, with a mean age of 47 years (range, 19-73) (Table 1).

- Mean follow-up of 6 years.

- Sixty-one CTGPI were performed on 46 patients (1 injection, 34 patients; 2 injections, 9 patients; and 3 injections, 3 patients).

\section{Outcomes:}

- We reviewed the ambulatory digital medical records to collect data on previous treatments, pain intensity at the time of the injection, and at the first postinjection follow-up 4 weeks after the procedure, or if the patient had had surgery.

- As microdiscectomy was considered a failure of CTGPI, the patients were not evaluated further following surgery regardless of the surgical result.

- Radicular and low back pain were independently evaluated according to the visual analogue scale (VAS), graded from 0 (no pain) to 10 (maximum pain ever suffered).

- We also documented whether the procedure had been painful and how many days later the decrease in pain was noticed and possible complications.

- For the final follow-up evaluation at time of the study review, all patients were contacted by a physician not directly involved in their treatment (Table 3). Patients gave oral consent but not written consent for the study.

- We also documented when the patient was able to resume work and sports activities.

- In all cases, the diagnosis of foraminal or extraforaminal disc herniation was performed by magnetic resonance imaging according to Mc Culloch [1].

\section{Analysis:}

- The statistical analysis utilized for VAS values was the Wilcoxon signed rank test for paired samples.

Additional methodological and technical details are provided in the web appendix at www. aospine.orglebsj. 
Fig 1 Patient sampling and selection.

\section{Total patients receiving intervention during time period} $(\mathrm{N}=1542)$

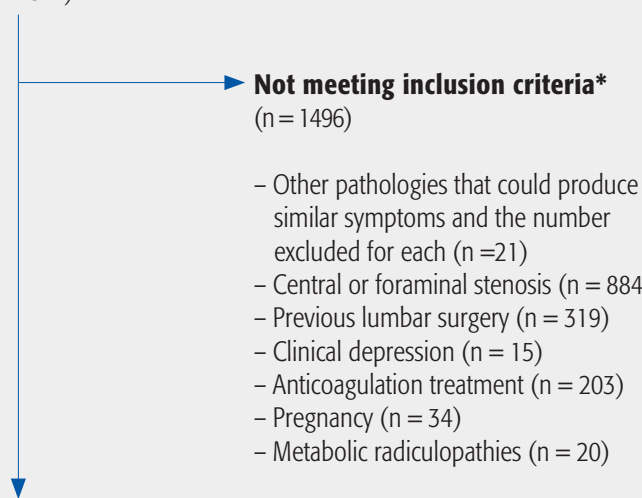

Eligible

$(n=46)$

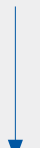

Enrolled

$(n=46)$

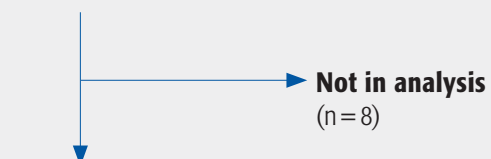

Patients available

for analysis

$(\mathrm{n}=38)$

- Other lost to follow-up $(n=0)$
- Patients with less than 2 years of data $(\mathrm{n}=0)$

- Death $(n=0)$

- Patients who required surgery $(n=8)$
Table 1 Demographic and baseline characteristics of intervention groups.*

\begin{tabular}{ll}
\hline & $\begin{array}{l}\text { treatment } \\
\mathbf{n = 4 6 ,} \text { No. (\%) }\end{array}$ \\
\hline Age, y, mean \pm SD & $47.5 \pm 12.8$ \\
\hline Female & $21(46)$ \\
\hline Loss to follow-up & 0 \\
\hline Level injected L5-S1 & $13(28)$ \\
\hline Level injected L4-L5 & $24(52)$ \\
\hline Level injected L3-L4 & $8(17)$ \\
\hline Level injected L2-L3 & $1(3)$ \\
* $P$ values for between-group differences as determined by 2-sampled \\
$t$ test for continuous measures and $\chi^{2}$ test for proportions.
\end{tabular}

Table 2 Outcomes being studied.*

\begin{tabular}{llll}
\hline outcome & $\begin{array}{l}\mathbf{N}=\mathbf{3 8} \\
\text { (before treatment) }\end{array}$ & $\begin{array}{l}\mathbf{N}=\mathbf{3 8} \\
\text { (after treatment) }\end{array}$ & $\boldsymbol{P}$ value \\
\hline $\begin{array}{l}\text { VAS low back } \\
\text { pain, mean } \pm \text { SD }\end{array}$ & $7.6 \pm 2.2$ & $2.3 \pm 2.6$ & $<.0005$ \\
\hline $\begin{array}{l}\text { VAS radicular } \\
\text { pain, mean } \pm \text { SD }\end{array}$ & $7.6 \pm 2.6$ & $0.5 \pm 1.7$ & $<.0005$ \\
\hline
\end{tabular}

* VAS indicates visual analogue scale. $P$ was obtained using the Wilcoxon signed rank test for paired samples. 


\section{RESULTS}

- Of 46 patients with a foraminal or extraforaminal disc herniation treated with CTGPI, all surgical candidates, $8(17 \%)$ were finally operated.

- Among 38 patients who did not require surgical treatment, 28 (74\%) improved within the first 15 days.

- At 1-month postinjection, 41 (89\%) patients stated a decrease in radicular pain intensity: 3 , no change; and 2 received surgical treatment.

- The final evaluation showed that $38(83 \%)$ patients had not required surgery. Compared with pain levels at the preinjection examination, low back pain had decreased a mean of 5.3 points (from 7.6-2.3) and radicular pain had diminished a mean of 7.1 points (from 7.6-0.5) in this group, $P=.001$.

- Twenty-two (58\%) of 38 nonoperated patients referred no pain at all.

- Thirty-five patients who had not required surgery had resumed their normal everyday activities (See web appendix for complete patient series.)

- No complications that could be directly related to the procedure (infection, headache, bleeding, and adverse reactions to the medication requiring further treatment) were recorded.

\section{DISCUSSION}

- We first reported our results with CTGPI in 1996 [2]; our impression was that this procedure was effective for the management of patients with severe radicular pain due to disc herniation.

- We included only patients with foraminal or extraforaminal disc herniation because the natural history of disc herniation in this location is less favorable $[3,4]$

- Of 46 patients, who were all deemed surgical candidates, 8 (17\%) were finally operated.

- Among 38 patients who did not require surgical treatment, 28 (74\%) improved within the first 15 days. These findings, in patients who had gone through several weeks of pain and multiple treatments, make it unlikely that pain improvement had followed the natural history of the disease (6-12 months).

- The documented pain score difference before CTGPI and at final follow-up as expressed with a visual analogue score seems to recall the natural evolution of disc herniation.

\section{Strengths:}

- We only evaluated lateral herniation.

- Our cohort was assembled from a community clinical practice, making it likely that our results are broadly generalizable.

\section{Limitations:}

- Relatively small number of patients included.

- Retrospective study without a control group. However, our results are comparable with other studies that are prospective [5].

- In the two most frequently cited prospective randomized double-blinded studies, diverse results are reported.

- After reviewing the available literature, it becomes obvious that there are no perfect protocols to show the efficacy of nerve root injection (NRI). Even surgical candidates in control groups avoid surgery in $33 \%-48 \%$.

- Karppinen et al [6] found significant differences on almost every parameter between the study group (bupivacaine plus methylprednisolone) and the control group (saline) only at the 2 -week follow-up. This implies that the procedure only brings short-term clinical benefit. However, $23 \%$ of their population has normal or bulging discs, which we consider not surgical candidates in our practice.

- Riew et al [7] found that in their study group most patients with lumbar radicular pain who avoid an operation for at least 1 year continue to avoid operative intervention for a minimum of 5 years.

\section{Clinical relevance and impact}

- The natural history in patients with sciatica caused by a disc herniation is favorable [8-14], which explains why the effectiveness of CTGPI or any other procedure is difficult to establish.

- Epidural steroid infiltration under image intensifier guidance is not a new procedure and has been performed for more than 35 years [15]. However, its effectiveness is still controversial; while some reports demonstrate it as a useful alternative [8, 16, 17], others only compare its action with that of intramuscular administration or with the natural history of the disease [18-20].

- The use of CT guidance can make the procedure more expensive, and most clinicians support the use of image intensifier. In our institution, one of five CT scanners is assigned only to invasive percutaneous procedures and is usually available for scheduling a 10-minute CTGPI. Despite the increased initial costs of $\mathrm{CT}$, it may lead to financial savings in comparison with image-intensifier guided operating-room CTGPI as is performed in many institutions; additionally, the image is biplanar instead of monoplanar and is more accurate. 


\section{SUMMARY AND CONCLUSIONS}

Based on our results, we consider that the use of CT-guided periradicular infiltration is a safe and reliable alternative for patients with foraminal or extraforaminal disc herniation without severe motor deficit that remain with intractable radicular pain after adequate conservative treatment.

\section{REFERENCES}

1. Mc Culloch JA (1991) The Adult Spine: Principles and Practice., New York: Rawen Press Ltd, 1821.

2. Ortolan E, Sola C, Gruenberg M, et al (1996) CT-guided periradicular corticosteroid injection. Presented at SICOT '96 Annual Meeting, August, 1996, Amsterdam, Holland.

3. Epstein NE (2002) Foraminal and far lateral lumbar disc herniations: surgical alternatives and outcome measures. Spinal Cord; 40(10):491-500.

4. Rust MS, Olivero WC (1999) Far-lateral disc herniations: the results of conservative management. J Spinal Disord; 12(2):138-140.

5. Riew KD, Yin Y, Gilula L, et al (2000) The effect of nerve-root injections on the need for operative treatment of lumbar radicular pain. J Bone Joint Surg; 82-A:1589-1593.

6. Karppinen J, Malmivaara A, Kurunlahti $M$, et al (2001) Periradicular infiltration for sciatica: a randomized controlled trial. Spine; 26:1059-1067.

7. Riew KD, Park JB, Cho YS, et al (2006) Nerve root blocks in the treatment of lumbar radicular pain: a minimum five-year follow-up. J Bone Joint Surg; 88(8):1722-1725.

8. Vad VB, Bhat AL, Lutz GE, et al (2002) Transforaminal epidural steroid injections in lumbosacral radiculopathy: a prospective randomized study. Spine (Phila Pa 1976); 27(1):1116.

9. Saal JA (1996) Natural history and nonoperative treatment of lumbar disc herniation. Spine; 21(24S):2-9.

10. Saal JA, Saal JS, Herzog RH (1990) The natural history of lumbar intervertebral disc extrusions treated nonoperatively. Spine (Phila Pa 1976); 15(7):683-686.

11. Roland M, Morris R (1983) A study of the natural history of back pain: part I. Development of a reliable and sensitive measure of disability in low-back pain. Spine; 8:141-144.

12. Taylor VM, Deyo RA, Cherkin DC, et al (1994) Low back pain hospitalization: recent United States trends and regional variations. Spine (Phila Pa 1976); 19(11):1207-1212.

13. Weber H (1983) Lumbar disc herniation: a controlled, prospective study with ten years of observation. Spine (Phila Pa 1976); 8(2):131-140.

14. Weber H (1994) The natural history of disc herniation and the influence of intervention. Spine (Phila Pa 1976); 19(19):2234-2238.

15. Macnab I (1971) Negative disc exploration: an analysis of the causes of nerve-root involvement in sixty-eight patients. J Bone Joint Surg Am; 53(5):891-903.

16. Bush K, Hillier S (1991) A controlled study of caudal epidural injections of triamcinolone plus procaine for the management of intractable sciatica. Spine (Phila Pa 1976); 16(5):572-575.

17. Dilke TF, Burry HC, Grahame R (1973) Extradural corticosteroid injection in management of lumbar nerve root compression. BMJ; 2(5867):635-637.

18. Koes BW, Scholten RJ, Mens JM, et al (1995) Efficacy of epidural steroid injections for lowback pain and sciatica: a systematic review of randomized clinical trials. Pain; 63(3):279288.

19. Carette S, Leclaire R, Marcoux S, et al (1997) Epidural corticosteroid injections for sciatica due to herniated nucleus pulposus. $N$ Engl J Med; 336(23):1634-1640.

20. Cuckler JM, Bernini PA, Wiesel SW, et al (1985) The use of epidural steroids in the treatment of lumbar radicular pain: a prospective, randomized, doubleblind study. J Bone Joint Surg; 67A:63-66. 


\section{EDITORIAL PERSPECTIVE}

The reviewers congratulate the authors on taking on this contentious topic of managing lumbar disc herniation with nonsurgical care and openly discussing the shortcomings (retrospective, absence of comparison group, reliance on a single score only -VAS, no ODI, EQ-5D, SF-12, or others were used, and the natural course history of foraminal disc herniation remains unknown). The authors very convincingly showed a large percentage of their patients experiencing dramatic pain decrease through high-quality foraminal injections guided by CT scan imaging in the hands of seasoned interventionalists. The use of this imaging modality and the apparent quality of the injection specialists at the study site present a clear difference to the methods presented by authors of other studies, such as the classic study by Cuckler et al [1].

The topic of epidural injections and their efficacy and efficiency has eluded conclusive answers from formal prospectively randomized trials for some time now. The dynamic nature of disc herniation in particular has been a similar source of frustration for organizers of much larger well-funded prospective trials, such as the SPORT trial with 1092 patient [2].

The number and dimensions of confounding variables continue to pose prohibitive impediments for these studies, with issues including disc pathology (size and location of disc herniation, pressure and/or tension created on neural elements), patient factors (age, neural status, pain tolerance, comorbidities, and baseline functional status to name a few) and many other factors, such as duration of symptoms, expectations, and patient preferences all playing a role in the final symptom presentation. Attempts at defining the natural course history of radiculopathy, beyond stating the obvious, have been frustrating as well; basically, most patients with radiculopathy get better on their own [3].

The role of interventions, such as discussed in the article by Gruenberg et al, remains unclear. Do they intend to (1) decrease the utilization of surgical decompression (surgery seen as failure of nonoperative care); (2) shorten the duration of the natural course history of patients with radiculopathy (treatment effect); or, (3) merely attenuate patient discomfort during the acute phase? Also, how soon upon initial presentation of a patient with radicular symptoms does one recommend injection? Right away or should one wait for some time? Then there is the question of repeated injections. How long should patients expect to have pain relief with such an injection? When and how often should one inject again? What accompanying protocol should a patient follow? Lead a normal life or pursue some form of a special regimen? This is a complex web of issues without clear metrics for some of the concerns raised, most of all the underlying question of quantifying the actual duration of the natural course history of symptomatic disc herniation, which has been described to range from a few days to weeks or even months.

Another important variable difficult to distinguish is that of surgeon threshold for procedures and the 'treatment culture' present in any given region or country. There are undoubtedly different expectations set by initial practitioner behavior regarding patients presenting with radiculopathy. The expectations set by practitioners and the overall healthcare culture likely influence patient behavior dramatically - setting the stage for patients requesting early surgery out of fear of neurological deterioration and with the promise of earlier return to a normal life compared with those being willing to put up with nonoperative care for radiculopathy without motor deficit even without getting an MRI scan for weeks or months [4]. The variability of physician behaviors, range of responses, and individuality of care will inevitably clash with insights gained through review of larger patient databases, such as collected in registries.

For now, this study suggests that a well-performed foraminal epidural steroid injection is a treatment option for patients with radicular symptoms. When considering future studies involving epidural steroid injections the possibility of including CT-based confirmation of periradicular needle location for patients with selected pathology certainly would seem to be a worthwhile consideration.

1. Cuckler JM, Bernini PA, Wiesel SW, et al (1985) The use of epidural steroids in the treatment of lumbar radicular pain: a prospective, randomized, doubleblind study. $J$ Bone Joint Surg; 67-A:63-66.

2. Pearson A, Lurie J, Tosteson T, et al (2011) Who should have surgery for an intervertebral disc herniation? Comparative effectiveness evidence from SPORT. Spine (Phila Pa 1976); 2011 June 15. [Epub ahead of print].

3. Casey E (2011) Natural history of radiculopathy. Phys Med Rehabil Clin N Am; 22(1):1-5. Epub 2010 Dec 3.

4. Gremeaux V, Viviez T, Bousquet $\mathbf{P}$, et al (2011) How do general practitioners assess low back pain websites? Spine (Phila Pa 1976); 2011 Mar 14. [Epub ahead of print]. 